\title{
Brick-Building Interface Support for Cocreative Communication
}

\author{
Shigeru Wesugi \\ Consolidated Research Institute for Advanced Science and Medical Care \\ Waseda University \\ Yoshiyuki Miwa \\ School of Science and Engineering, Waseda University
}

\begin{abstract}
To support cocreative communication between people who are in separate, remote locations, embodied communication is crucial and a common place for communication should be established. This article proposes an idea to integrate remote, physically restricted places into a shared virtual space to bridge these remote places and to describe interface systems for cocreative communication. To show a typical example of cocreative communication with a high degree of freedom for bodily actions, this study focused on brick-building play, in which people are free to construct structures spatially with physical objects. For remote collaborative communication including modeling with physical bricks at each physical place, two interface systems were designed: the brick-modeling interface system and the brick-reader glove interface system. The brick-modeling interface system makes it possible for users to act out others' brick plays with physical bricks in the remote places and in shared virtual space in real time. The brick-reader glove interface system enables users to act out the modeling process with virtual bricks and virtual avatars of both remote users in the shared virtual space. The experiment clearly suggests that these interface systems are useful for creating a shared virtual space and for supporting collaborative work in three dimensions with a sense that the remote people were together in the same place.
\end{abstract}

\footnotetext{
A publication of the Human Interface Society

The authors thank Shiroh Itai, Tetsuro Numata, and Soichiro Atsumi who have cooperated to this research. We would also like to thank Dr. Katsunori Shimohara and Dr. Yoshiki Oshida for their help in editing the final version of this article.

Correspondence should be addressed to Shigeru Wesugi, 41-5-2-203, 17, Kikui-Cho, Shinjuku-ku, Tokyo, Japan 162-0044. E-mail: wesugi@aoni.waseda.jp
} 


\section{INTRODUCTION}

People experience physical encounters with other people when sharing the same physical space, and they convey their thoughts and emotions through not only verbal communication but also bodily expressions, including facial expression, eye gaze, and gesture. Individuals can also attune themselves to mood and identify with each other through a series of embodied interactions. Interacting physically in this way is a crucial part of everyday human encounters (Dreyfus, 2001; Miwa, 2001; Shimizu, Kume, Miwa, \& Miyake, 2000). Human-human communication in a physically present situation, mutually creating and sharing context through embodied interactions, is called cocreative communication (Miwa, 2001; Shimizu et al., 2000), which can be seen in various social situations from everyday activities in a local community to a development process for a new product.

To support cocreative communication between people in remote places, a physically present situation, or "just now, here" (referring to when individuals should interact and where they exist), should be shared among communicators, even if they are actually physically separated from each other (Miwa, 2001; Shimizu et al., 2000). Creation of this shared situation is fundamental to interpersonal collaborative communication (Miwa, 2001; Shimizu et al., 2000).

On the other hand, many messages can be transmitted "anytime and anywhere" these days using telecommunications like mobile phones and e-mail. However, current computer-supported collaborative work tools in telecommunications still have a drawback: A sense of spatial and temporal presence cannot be shared among remote people (Dreyfus, 2001).

Because of these aforementioned issues, we have been engaged in an ongoing research project to support cocreative communication, particularly focusing on problems of embodiment and bodily awareness (a sense of bodily actions and indications of facial expression) in a virtual space (Miwa, 2001; Miwa, Wesugi, Ishibiki, \& Itai, 2001; Wesugi, Itai, \& Miwa, 2001).

In this article, we propose the integration of remote, physically restricted locations into a shared virtual space to bridge these remote places, and we describe interface systems for cocreative communication. To show a typical example of cocreative communication with a high degree of freedom of bodily actions, this study employs brick-building play, in which people are free to construct structures spatially with physical objects. Group play, such as brick-building play and sandbox play, is expected to cultivate sociality in childhood (Kasama, 2001). In addition, theimportance of brick-building play has been demonstrated in its potential as a tool for seniors in the rehabilitation of their hand and arm functions (Naef \& Kashiwagi, 2000).

To support brick-building play as social and physical activities between remote people, we have devised and implemented two interface systems that enable users to create model structures with physical bricks in their real space and to act out the modeling process in the shared virtual space with virtual bricks and virtual avatars. The experimental results clearly suggest that these interface systems can work well, creating a shared virtual space representing the visual constructing process with physical bricks and supporting collaborative work in three dimensions, giving people the sense that they are in the same place as the other, remote people. 


\section{BRICK-BUILDING PLAY BETWEEN REMOTE PLACES}

\subsection{Communication of Brick-Building Play}

As the first step in supporting brick-building play between remote people, it is necessary to investigate what bodily interactions should be taken into consideration and to analyze bodily actions during group brick-building play. Focusing on sharing a body-aware space at each real place, we propose a novel approach to supporting collaborative modeling with physical bricks among people who are in separate places.

Figure 1 shows a conceptual image of cocreative communication in the brick-building play (and sandbox play) between remote places. Through sharing a virtual space, a user can pick up, raise, pile up, and take away a brick as individual actions, and the user can pass a brick to others and pile up bricks as a collaborative action with a group.

We classified complex bodily actions in the brick-building play into two modes: handling a physical brick directly and nontouching any physical brick. These two actions are included in the handling mode of a physical brick. The mode of nontouching any brick includes gestures such as pointing at a brick and/or its position and conversation with another person for collaboration.

Each individual should be able to do these actions naturally in the common shared space integrating the remote places. To support brick-building play between remote places, the three requirements for a system should be as follows:

1. Allow people to manipulate a physical brick intuitively and spatially at each physically real place.

2. Represent the individuals' bodily actions such as pointing, directing, and other spatial expressions shown in their collaboration.

3. Allow people to interact virtually with any physical brick at the other remote place.

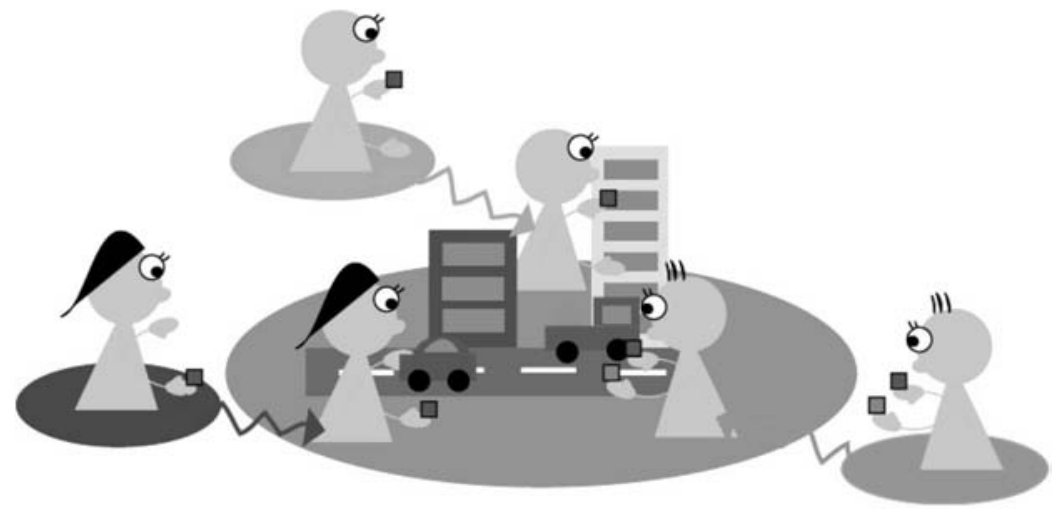

FIGURE 1 A conceptual image of cocreative communication in the brick-building play between remote places. 
In this article, we aim to achieve Requirements 1 and 2 as the first approach in supporting group brick-building play between remote places, and we propose a novel design approach for interface systems for that purpose in the next section.

\subsection{Interreal Virtual Space Integrating Remote Real Places}

A useful method to employ is sharing a video of modeling with physical bricks with each other in a real place. However, only sharing video cannot support remote collaborative communication including spatial expression such as indicating an object at a remote site (Heath \& Luff, 1991).

Instead, we propose a novel method to create a virtual space reflecting a situation for modeling in each physical place, integrating those virtual spaces into a common virtual space, and sharing the integrated virtual space with each remote communication user. Figure 2 illustrates the interface design method employed. A structure of physical bricks and bodily actions such as manipulating physical bricks in each physical place are represented visually in a virtual space. Those virtual spaces are then integrated into a common virtual space so that a spatial relationship among bricks and bodily actions in each virtual space is consistent in the same coordinate system. People can see the integrated virtual space from each viewpoint through head-mounted display (HMD), cathode-ray tube (CRT) display, and a video projected on a screen. We term the shared virtual space an interreal virtual space, because the integrated virtual space bridges the remote real places.
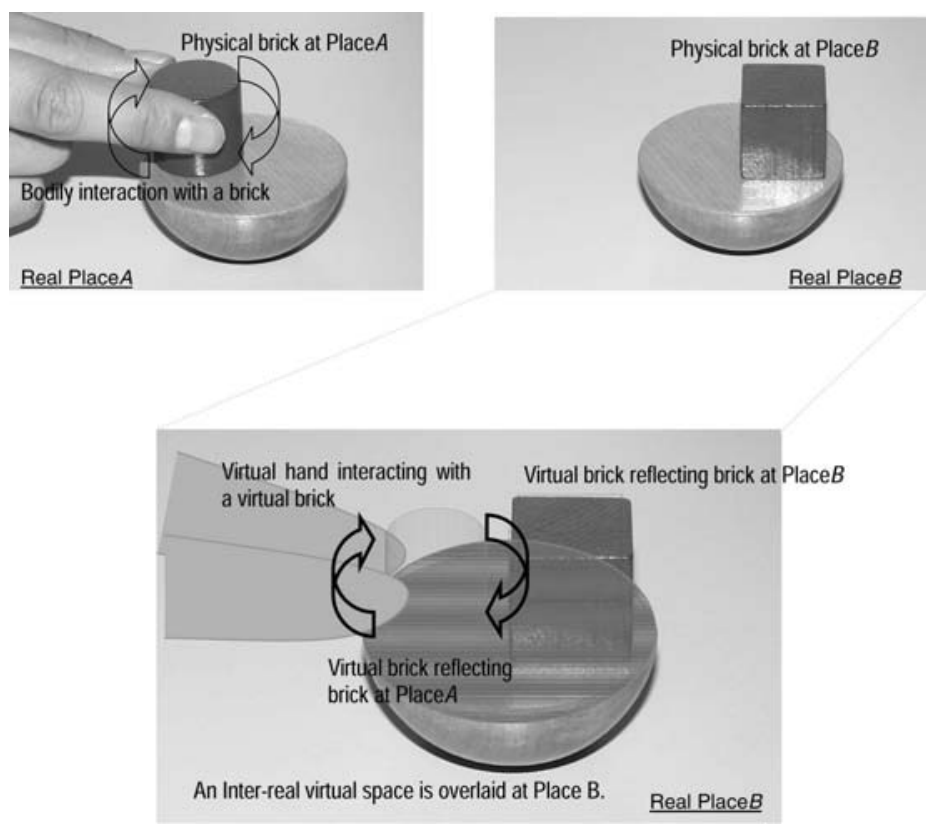

FIGURE 2 Design method of sharing an interreal virtual space between remote places. 
We devised two interface systems based on the aforementioned design approach. In Section 3 we describe a brick-modeling interface system to represent a virtual structure (constructed with physical bricks in the physical, real place) in a virtual space in real time. In Section 4 we describe a brick-reader glove system to represent the modeling process in a shared virtual space with virtual bricks and virtual avatars.

\section{BRICK-MODELING INTERFACE}

\subsection{Design}

We designed and implemented a brick-modeling interface system to represent a structure with physical bricks in a virtual space to satisfy Requirement 1 . To represent a virtual brick structure, the location, posture, shape, and color of the physical brick structure should be measured in real time. Thus, two methods can be suggested. First, each physical brick transmits its location and shape data to a host computer, and then the host computer visually reconstructs the structure based on the data set. Second, a CCD camera takes video of the structure with physical bricks, and a host computer analyzes the structure in three dimensions from its video. We chose the first method because of the independence of the computer performance and design architecture of the interface system representing a virtual structure. The virtual structure can be constructed based on the 3-byte structure data at one brick-brick connection in our architecture, as seen in Figure 3 . The structure data are composed of three scenarios: connecting brick ID (S-ID), connected brick ID (M-ID), and connecting situation (L-ID) between connected brick and connecting brick.

Figure 3 illustrates brick-assembling data. When a brick is connected to another brick, M-ID, S-ID, and L-ID are transmitted to a host computer as a packet. When a brick is disconnected from another brick, M-ID, null ID, and L-ID are transmitted to the host computer. Because the amount of brick-brick and brick-computer communication data and storage data in the computer are rela-

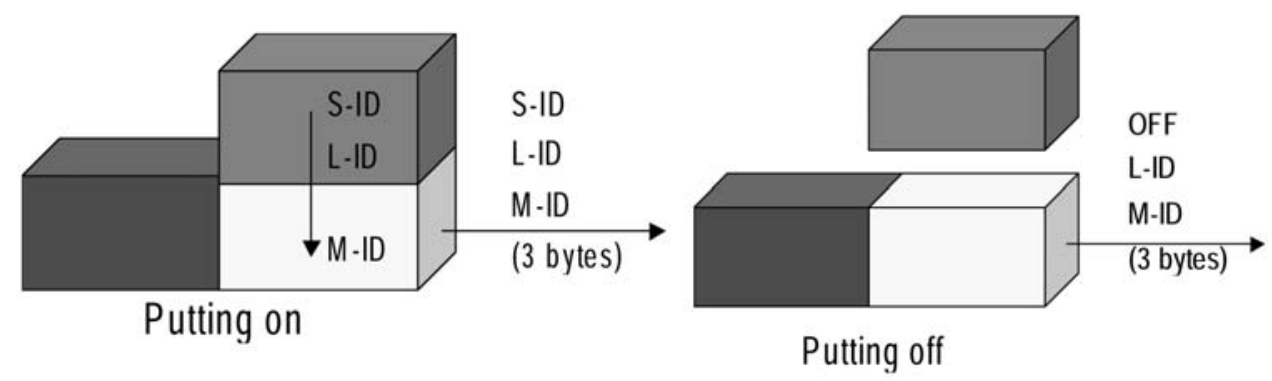

FIGURE 3 Three-byte structure data at one brick-brick connection in our unique architecture. S-ID = connecting brick; L-ID = connecting situation; $\mathrm{M}-\mathrm{ID}=$ connected brick. 
tively low, this method will reconstruct virtual structure in real time independent of the computer performance.

To implement a brick interface system based on this architecture, a brick itself should have three functions: data communication, electrical and mechanical connection, and sensing.

1. The communicating function is for transmitting data signals from a brick to another brick and from a brick to a host computer.

2. The connecting function is the electrical connection for transmitting data signals, and the mechanical connection is for joining bricks with each other.

3. The sensing function is for detecting location of the other connected brick and an on-off connection situation.

We propose a rectangular, solid brick equipped with these three functions: (a) The brick includes pin-type connectors on the top, which have a connecting function and a sensing function; (b) the signals of structure data transmit from a brick to a brick and a host computer through these connectors; and (c) the brick can be connected with another brick in various ways, as demonstrated in Parts a, b, and c in Figure 4.

We also propose that the luminance of a brick should be adjustable to solve an occlusion problem when people see a virtual brick that can be overlaid onto a physical brick through the optical see-through HMD. In addition, a brick should include actuators that indicate a touch by a remote user.

Various interface systems using physical objects have been proposed. For example, Triangles (Gorbet, Orth, \& Ishii, 1998) and ActiveCube (Kitamura, Itoh, Masaki, \& Kishino, 2000) demonstrate model structures in a virtual space. Cypher (Nakai, Tadenuma, Tanaka, \& Nakao, 2001) and 3D-AR (Poupyrev et al., 2002) arrange virtual objects by using physical objects. However, these systems are available only for solo use or a colocated situation. Therefore, no interface system has yet been proposed that supports remote communication including modeling with physical objects in a shared virtual space that integrates remote places.

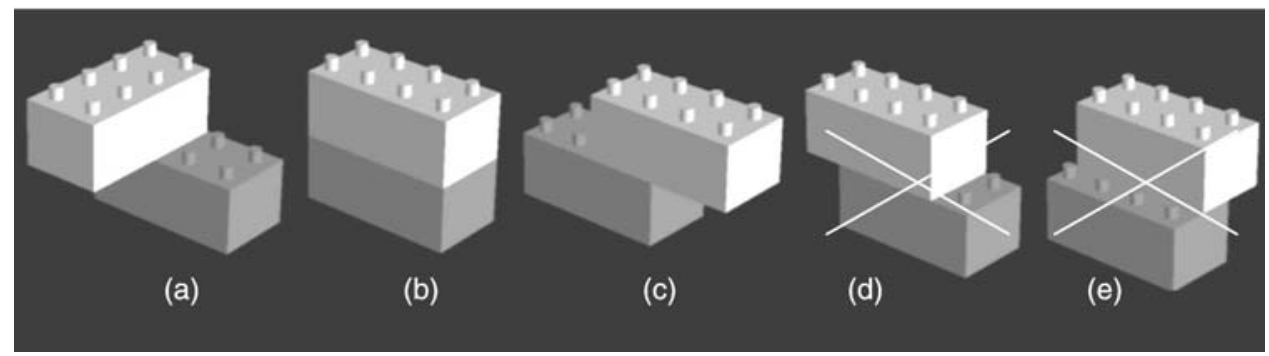

FIGURE 4 Various connections between two bricks. 


\subsection{Implementation}

The brick-modeling interface system is composed of three parts: a brick I/O device, a host computer for virtual brick generation, and an optical see-through HMD, as shown in Figures 5 and 6.

The brick I/O device consists of a basic brick unit and base brick unit, as shown in Figure 7. The basic brick unit, which can be manipulated by one hand, is a $144 \times$ $72 \times 60 \mathrm{~mm}$ and $500 \mathrm{~g}$ rectangular parallelepiped that has a $9 \mathrm{~V}$ and $1.5 \mathrm{~V}$ battery inside. The base brick unit consists of 12 basic brick units and has three functions: to relay information among bricks and the host computer, provide a foundation on

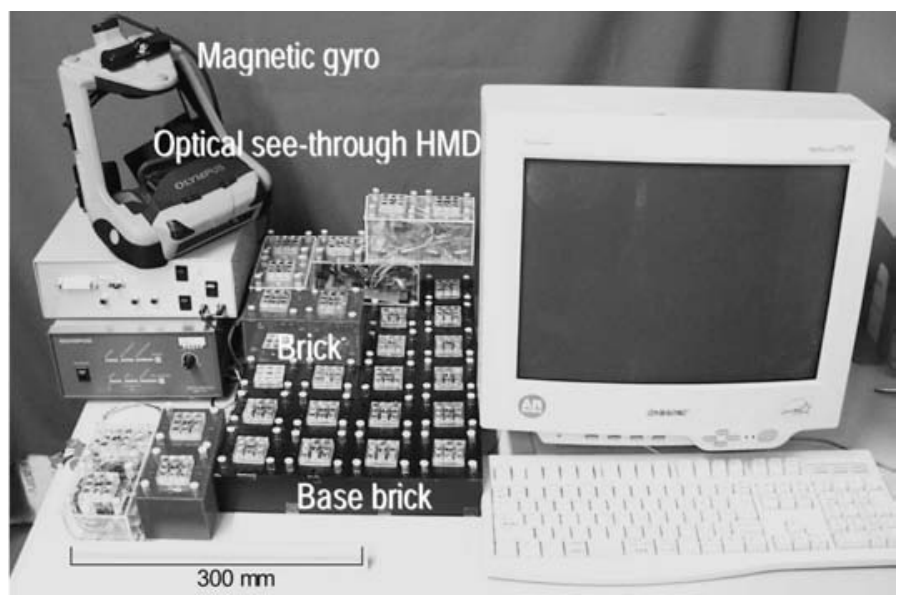

FIGURE 5 Brick-modeling interface system. HMD = head-mounted display.

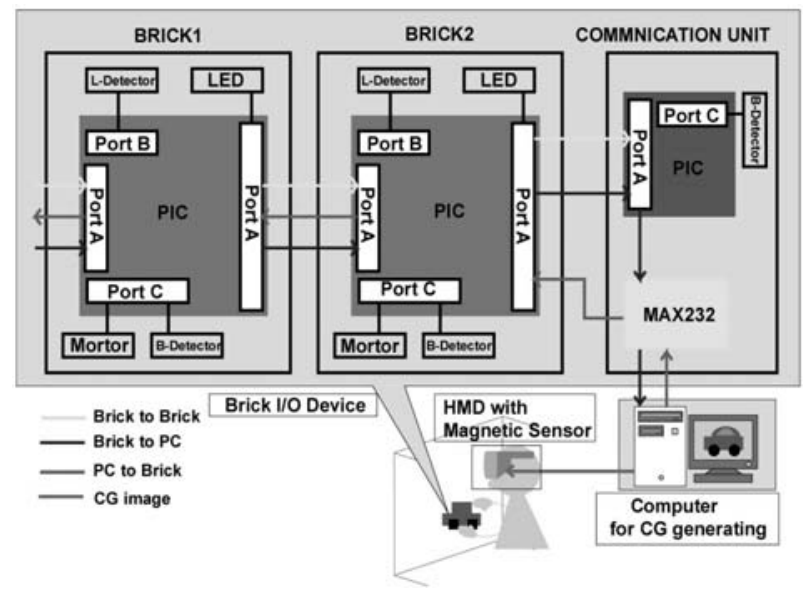

FIGURE 6 Schematic diagram of brick-modeling interface system. CG = computer graphic. 


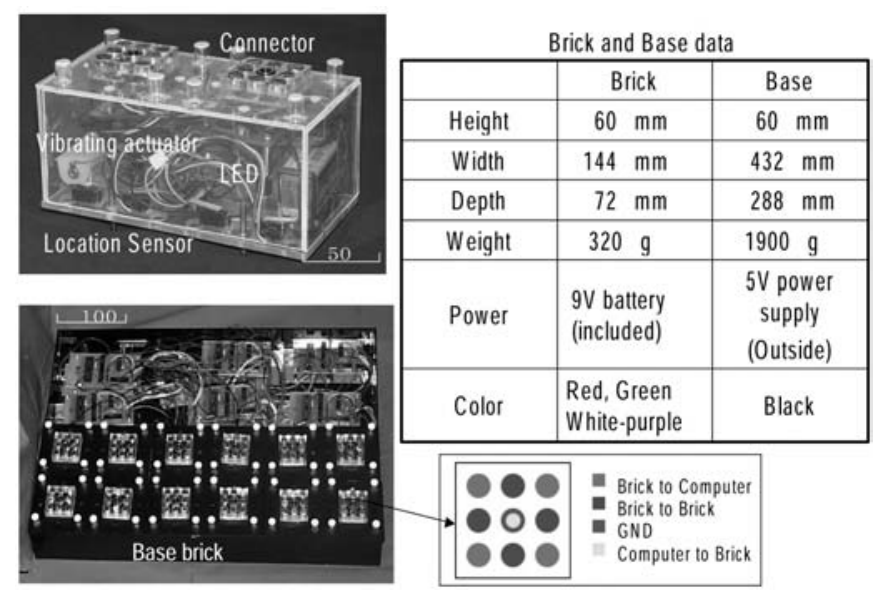

FIGURE 7 Basic brick unit and base brick unit. GND =

which a structure can be constructed, and provide sensing location of the brick on it. The power is supplied by an external source. The basic brick includes (a) eight sensors on its down side for detecting location of a connected brick and (b) a microcontroller (PIC16F873; Microchip Technology, Chandler, AZ) for processing data transmission. The brick can communicate with the host computer by 9600 bps serial communication. The brick has a connection-detecting sensor on the top for detecting connection with another brick to control data transmission to the host computer.

The connector is designed so that nine cupper coat magnets are located in a grid, as seen in Figure 7. Thus the same signal wire can always connect electrically even if the brick rotates by $90^{\circ}$. Figure 8 shows a way to detect the location of a connected brick. Three magnet pins and five nonmagnet pins are located on the top of the brick, and eight detectors are embedded under the brick. Figure $8 \mathrm{c}$ illustrates that, when a brick connects to another brick, the pairing magnet pins and detectors uniquely indicate a relative position between that brick and the other connected brick.

A vibrating actuator embedded inside is controlled by signals from the host computer. As soon as a brick connects with another brick, a virtual brick appears in the virtual space, and the actuator in the brick vibrates. Through these visual and tactile expressions, a user can understand that brick connection signals can transmit to the host computer successfully. In addition, when a remote communication partner connects a brick with another brick, the local user is aware of its connection, because the actuator in the local brick adjoining the brick that the remote user manipulated in the virtual space also vibrates, along with the appearance of a virtual brick.

The host computer rebuilds a virtual brick structure on the virtual base brick unit, as shown in Figure 9. The representing virtual space software is constructed by utilizing the WorldToolkit ${ }^{\circledR}$ library (Sense8, San Rafael, CA). 


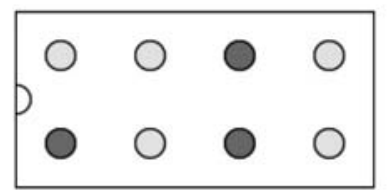

Top Face

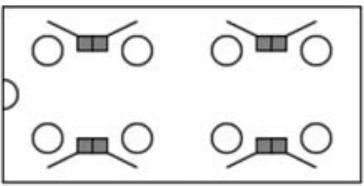

Bottom Face

$\bigcirc$ Pin Pin with Magnet

$\bigcirc$ Hole $\square$ Detector

(a) Allocation of sensor

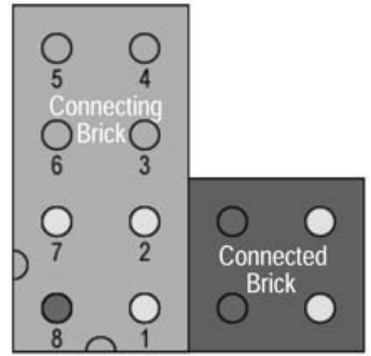

12345678

L L L L L L L H

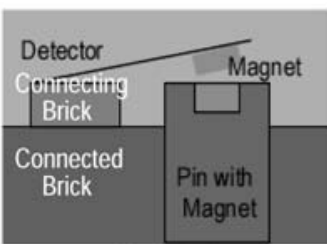

Detected

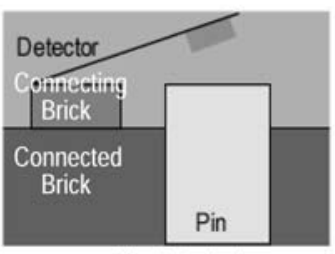

No Detected

(b) Mechanism of brick-connection sensor

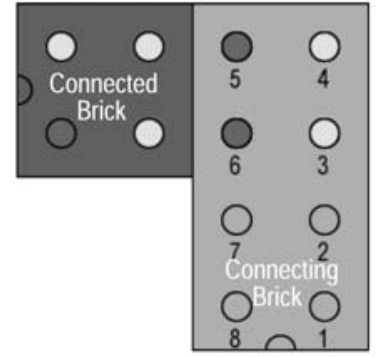

12345678

L L L L H L L

(c) Examples of the pairing connected among magnet pins and detectors for sensing position of connected brick

FIGURE 8 Detecting location of connected brick.

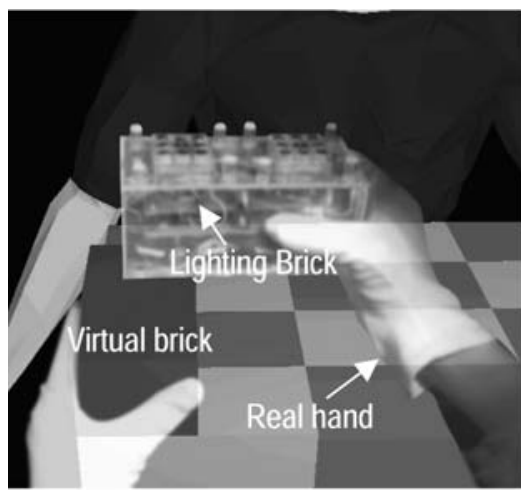

(a) Virtual modeling in the virutal space overlaid over the real place

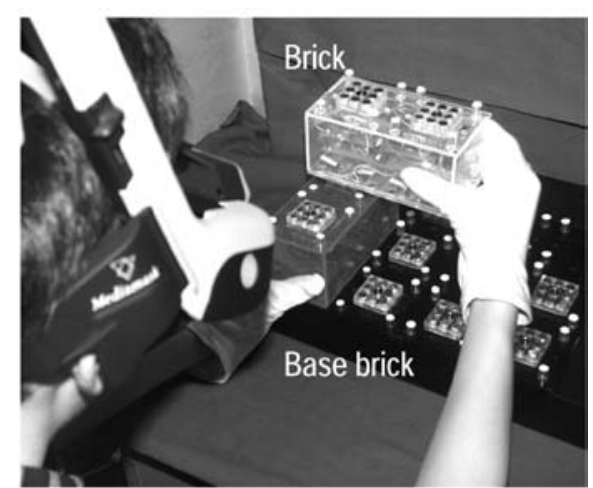

(b) Actual modeling with physical bricks

FIGURE 9 A scene of using the brick-modeling interface system. 
The virtual structure is overlaid in two dimensions onto the physical structure through the optical see-through HMD (Mediamask MW 601; Olympus, Tokyo, Japan). The registration is adjusted manually by data input via a keyboard.

Overlaying a virtual space onto the physical place causes an optical problem of occlusion between virtual bricks and physical bricks. We found that we could solve this problem by wearing a reflective glove and using its luminance, which increases more than that of a virtual brick. In addition, a user can identify a physical brick because an LED light is on before the connection is made and can identify a virtual brick because the LED is off when it is being connected.

\subsection{Evaluation}

At first, we investigated whether a structure with physical bricks can be visualized as a virtual structure in a virtual space in real time. Figure 10 shows a scene of a modeling process. We found that people can model with the brick device as easily as in usual brick-building play and a virtual structure can appear in real time. We also confirmed that a brick can connect with another brick in seven ways. These results clearly suggest that the brick interface system can produce a physical brick structure that represents a virtual structure in the virtual space in real time.

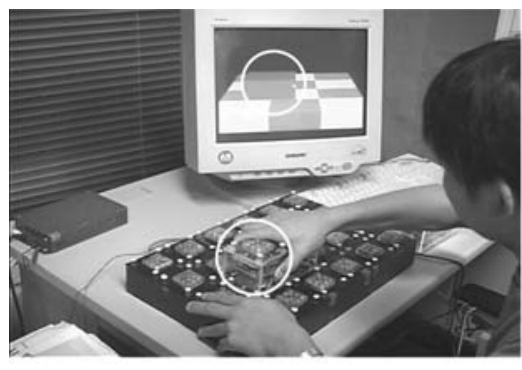

(a) User puts Blue brick on the Base brick.

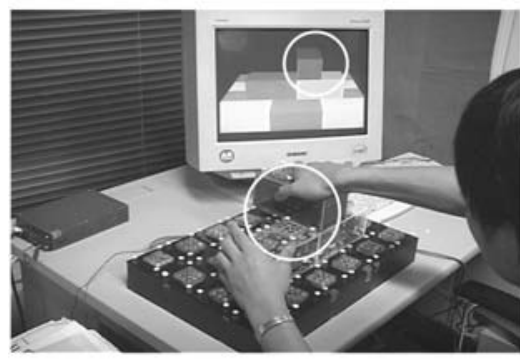

(c) User puts Red brick on the Green one.

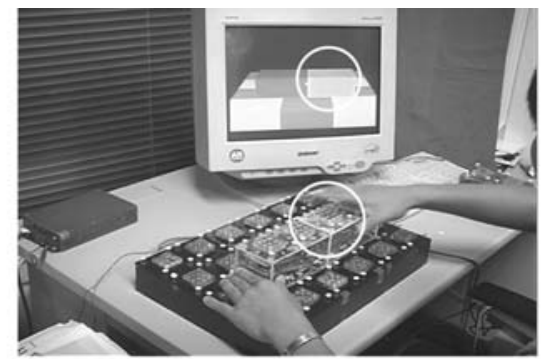

(b) User puts Green brick next to Blue one.

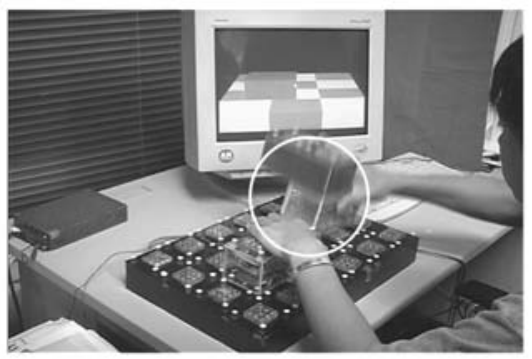

(d) User releases Red brick and Green one.

FIGURE 10 A scene of solo modeling. 
Furthermore, we developed the brick-modeling interface system to assist people who are physically separated in visually sharing each virtual structure in a common virtual space and investigated to what extent the system can support remote collaborative communication.

The brick interface systems, which are physically separated across two locations, are connected via a local area network. Two couples participated in the following experiment. Each couple was required to manually adjust the registration between a virtual brick, which is overlaid with the optical see-through HMD, and a physical brick. Then, both of them were asked to put the two colored bricks on and then take them away alternately without conversation. Figure 11 shows a scene of their modeling in utilizing the system. Each person can build a structure with physical bricks at each physical place and can share the virtual structure visually in the shared virtual space at the same time.

After the experiments, we briefly interviewed them on two questions: Can they can manipulate a brick intuitively with their own hands, and do they feel that they are modeling together?

Regarding the first question, most of the people pointed out that a brick cannot necessarily connect with another brick smoothly because the fit of the connector is firm. However, they also reported they could connect bricks with each other in the ordinary way after they became used to manipulating the brick. In response to the second question, some of them pointed out that it was hard to understand the intention of the other person without a conversation because a virtual brick just suddenly appears in the shared virtual space.

These results suggest that the brick-modeling interface system can represent a three-dimensional structure, constructed with physical bricks in each physical place, as a virtual structure in the shared virtual space.

\section{BRICK-READER GLOVE}

\subsection{Design}

To meet Requirement 2 as well as 1, bodily interactions with a physical brick and instruction to another person should be supported in a common communication place.

Tang's collaborative drawing research demonstrated the significance of sharing a working process and representing bodily actions, such as gesture in remote collaborative work (see Tang \& Leifer, 1988). Therefore, we proposed another design method to re-create a collaborative modeling process, which includes manipulating a physical brick and instructing other people with physical bricks in three dimensions in a shared virtual space.

We devised a novel brick-reader glove interface system, which creates a collaborative modeling process with physical bricks by utilizing a concurrent hand position and posture sensing system and a radio frequency identification (RFID) tag sensor, which has been used in the ubiquitous computing field (Rekimoto, Ullmer, \& Oba, 2001; Want, Fishkin, Harrison, \& Gujar, 1999). 

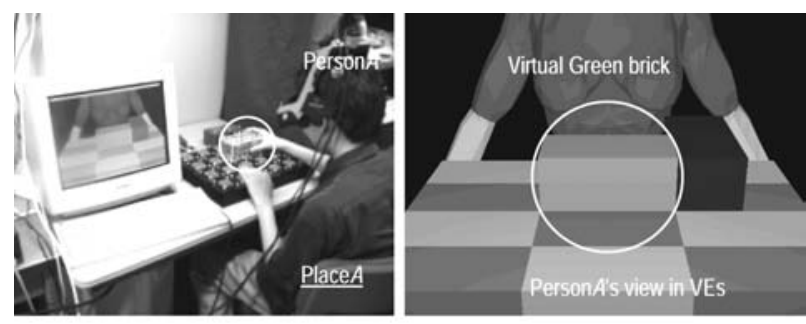

(a) Person $A$ puts Green brick on the Base brick.

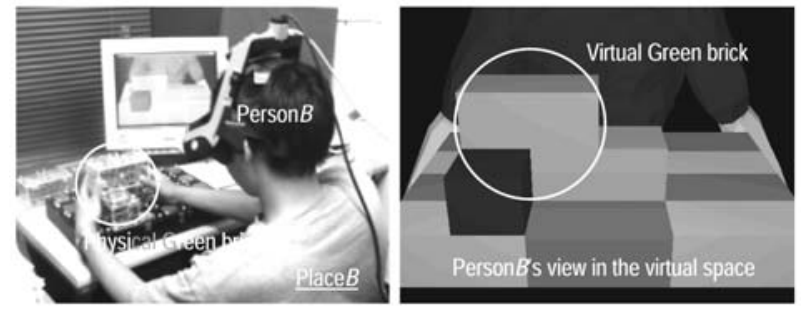

(b) Person $B$ puts Green brick on the Blue one.

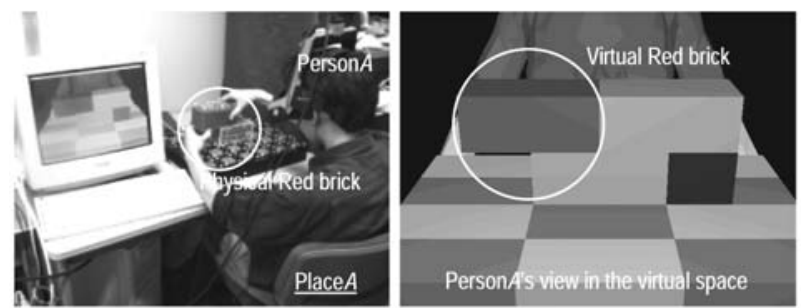

(c) PersonA puts Red brick on the Green one.

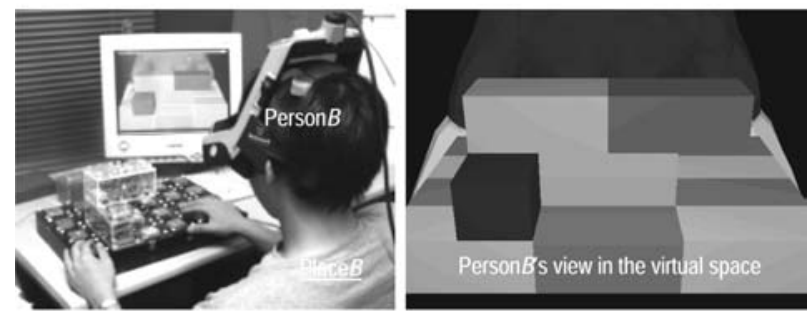

(d) Person $B$ sees the structure.

FIGURE 11 A scene of collaborative duet modeling.

\subsection{Implementation}

The brick-reader glove interface system is composed of five units, as shown in Figure 12: (a) a data glove for measuring finger flexure (5DT data Glove 5; Fifth Dimension Technologies, Irvine, CA); (b) a hand position and posture sensor that applies an electric field (Fastrak ${ }^{\circledR}$; Polhemus, Colchester, VT); (c) an RFID reader, an antenna $(40 \times 44 \mathrm{~mm})$ that is embedded in the data glove palm, and an RFID reader controller that is attached on the wrist (V720-HMC 73; OMRON, Tokyo, Japan ); (d) 


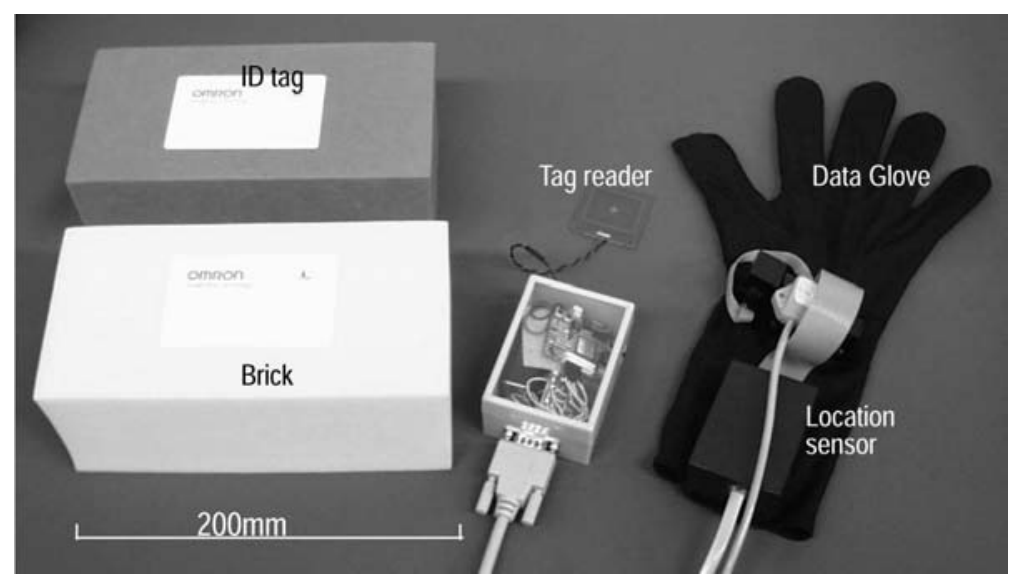

FIGURE 12 Brick-reader glove interface system.

a brick (polyethylene foam; $200 \times 100 \times 85 \mathrm{~mm}, 100 \mathrm{~g}$ ), on top of which an ID tag is attached $(85 \times 55 \mathrm{~mm})$; and (e) a host computer that receives data from the data glove, a position and posture sensor, and an RFID reader controller and that re-creates a virtual space based on those data. In addition, the host computer can communicate with a remote host computer to create a shared virtual space by transmitting the data just mentioned. At this time, the system is constructed for one hand only (the right hand).

First we explain an algorithm for re-creating a modeling process with a physical brick and the user's own physical hand in a virtual space, as shown in Figure 13.

1. A virtual hand appears and moves following a position and posture value $\mathrm{T}_{\mathrm{h}}\left(=\mathrm{a} \cdot \mathrm{T}_{\mathrm{H}}\right)$ in virtual-world coordinates $\mathrm{C}_{\mathrm{vw}}$. The coordinates $\mathrm{C}_{\mathrm{vw}}$ are calculated from $\mathrm{T}_{\mathrm{H}}$, which is measured by the position and posture sensor of the hand attached on the data glove in real-world coordinates $C_{\mathrm{RW}}$, where the brick is untouched.

2. When a user grasps a brick by his or her hand, an RFID antenna identifies the tag ID properties (color and shape), which are preregistered in a host computer, and the virtual brick appears with a virtual hand. The virtual brick is represented on the virtual hand by applying $\mathrm{F}_{\mathrm{b}}$, or positional relationship parameters, between the virtual brick and the virtual hand in virtual hand coordinates $C_{v h}$. The parameter $F_{b}$ is calculated from the grasping calibration mentioned later.

3. While the user transfers the physical brick by his or her hand, the positional relationship value remains $F_{b}$. The virtual hand holding the virtual brick is represented based on $\mathrm{T}_{\mathrm{h}}$ in $\mathrm{C}_{\mathrm{vw}}$.

4. At the moment the user's hand releases the physical brick, the virtual brick is positioned based on the hand-releasing position $\mathrm{T}_{\mathrm{h}^{\prime}}$. That is, the virtual brick is represented based on $T_{b}\left(=F_{b} \cdot T_{h^{\prime}}\right)$ in virtual-world coordinates $C_{v w}$.

As just described, a modeling process with physical movement of bricks by the user's own hand in a physical place is re-created visually in a virtual space. Then 


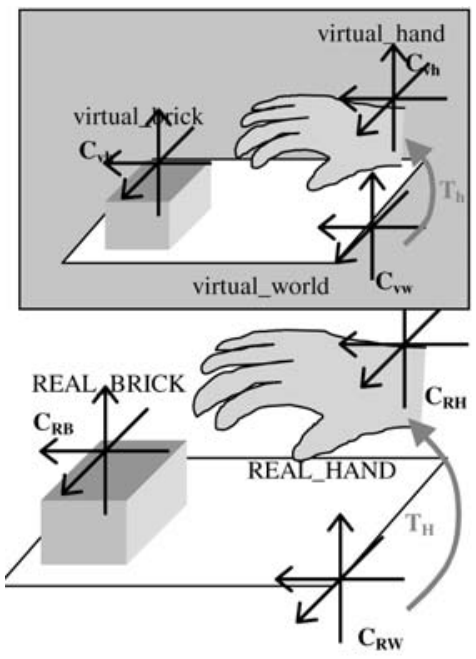

REAL_WORLD

(a) Going for

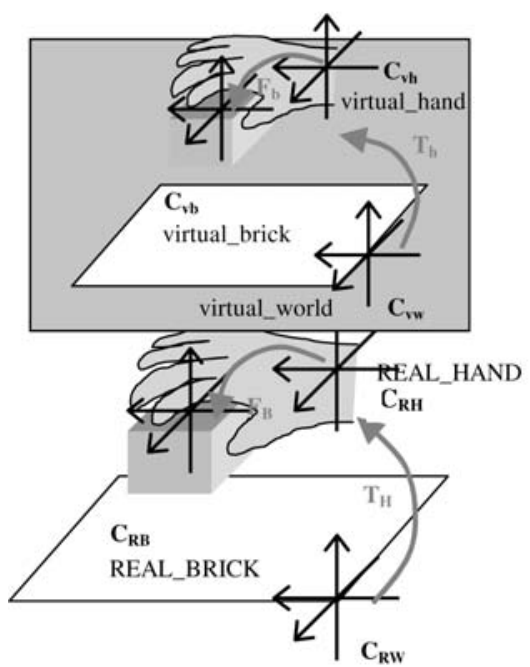

REAL_WORLD

(c) Hold
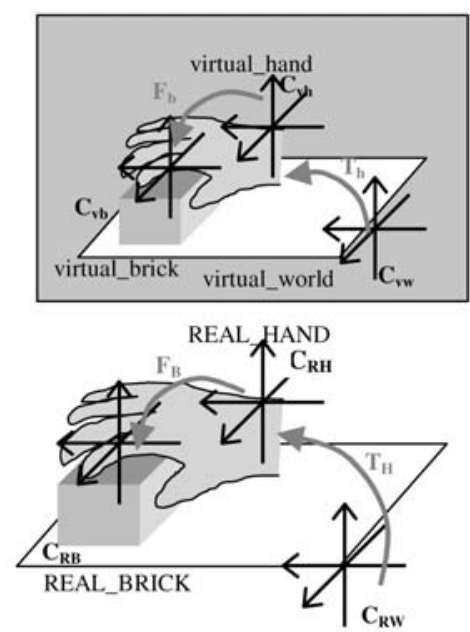

REAL_WORLD

(b) Grasped

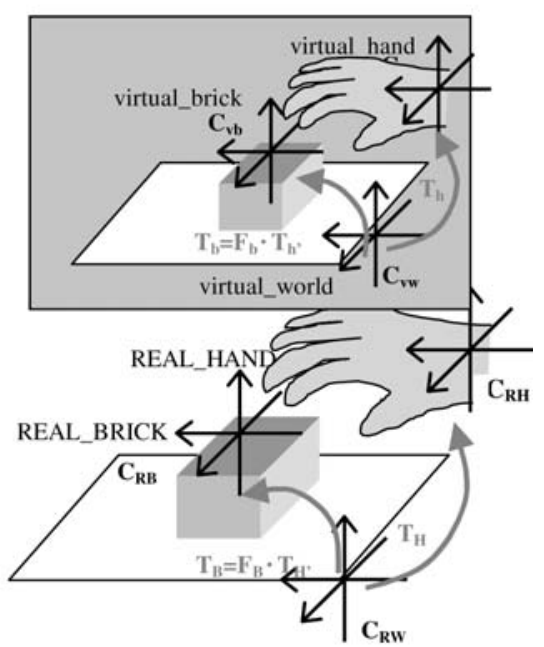

REAL_WORLD

(d) Released

FIGURE 13 Correspondence between virtual-world coordinates and real-world coordinates.

the virtual-modeling process at each site is integrated in a common virtual space. In addition, releasing a physical brick at any position can lead to the representation of a virtual brick on another virtual brick, which can be put on by a remote person.

The grasping calibration is required to adjust a positional relationship between the grasping virtual hand and the grasped virtual brick, because hand size and the 
position of grasping a physical brick vary from person to person. Before using this system, we calculated a parameter $\mathrm{F}_{\mathrm{b}}$ of rotation and translation of a virtual brick so that the relationship between position and posture of a physical hand and the physical brick, as a person grasps the physical brick, should agree with the relationship between position and posture of a virtual hand and the virtual brick.

This method of representing bodily interactions with physical objects in a virtual space is also applied in the Real Reality Modeling Interface (Schäfer, Brauer, \& Bruns, 1997). The interface system can recognize an interaction of grasping, transferring, and putting on an object located at the preregistered position by combining the data glove's open-closed condition with the position and posture of the hand. However, this system cannot distinguish grasping an object from just a bending hand, and it cannot identify the position of an object when the target object is located on the other object or when the position of the object at the preregistered position is moved accidentally.

In contrast, our brick-reader glove interface system can prevent misreading as just mentioned in the previous system, because the reader identifies the ID of the object whenever a user touches the object. In addition, the system is intended to support remote collaborative modeling in three dimensions in a shared virtual space integrating two remote places.

\subsection{Evaluation}

Position and posture data of a hand and a brick may include errors when a metal object, such as a coil, exists in the field, because the sensor system uses an electromagnetic field. To investigate the effects of a tag reader and an ID tag, the accuracy of the position of a hand with a brick is measured in the following way. Paper tapes with $1 \mathrm{~mm}$ scale are set along the XYZ supports of a wood table and are marked at intervals: X, $500 \mathrm{~mm} ; \mathrm{Y}, 400 \mathrm{~mm}$; Z, $300 \mathrm{~mm}$. Then the position of a hand grasping a brick was measured whenever it moved between two marks along each axis. The average of five experiments indicates that $X=499.9 \mathrm{~mm}(\mathrm{~s}=$ $0.951), Y=396.0 \mathrm{~mm}(\mathrm{~s}=0.729)$, and $\mathrm{Z}=302.7 \mathrm{~mm}(\mathrm{~s}=2.075)$. The range of errors is sufficiently narrow by approximately $7 \%$ when compared to the shortest side of the brick. Consequently, adverse influences of the RFID antenna and ID tag can be disregarded.

Solo modeling. To investigate how users model a structure with physical bricks in a virtual space when they utilize the brick-reader glove interface system, we conducted experiments on modeling with four participants. After the grasping calibration, they were asked to freely transfer two physical bricks and put one brick on the other brick, with one hand repeatedly in front of a CRT display. After all experiments, we reviewed the videos that recorded the modeling process.

Figure 14 shows a scene of modeling utilizing the system. Each picture in Figure 14 indicates scenes of grasping, moving, and releasing a brick in a virtual space as well as in a physical place corresponding to a modeling process, as in Figure 13. 
We found that the users regrasped and re-placed a physical brick while seeing a virtual brick on the display when the virtual brick could not appear in the virtual space. This action was taken because (a) the users grasped a part of the brick not attached to the ID tag or (b) the position of a virtual brick was different from that of a physical brick. It was observed the users would regrasp and re-place right at the beginning of their getting used to this system. Some of the participants also pointed out that they needed to acquaint themselves with how to grasp and release a brick using the brick-reader glove system.

The experiments suggested that the system can represent a modeling process with physical bricks in three dimensions and in real time in a virtual space, but users may require some training on how to interact with a brick.

Multiple modeling. This study investigated how two users located in physically separate places can model collaboratively through a shared virtual space using structures with physical bricks. Experiments on modeling were conducted using the brick-reader glove interface system installed at two separate places.

The participants were asked to freely and collaboratively construct structures with physical bricks using conversation. They could see a shared virtual space projected on the screen in front of them from each viewpoint fixed at that time. Participants conducted the grasping calibration at each place before the experiments.

After all the experiments, we held a brief interview with the users and asked them to what extent they could freely construct a structure with the system and how they could model structures collaboratively with a remote communication partner. We also reviewed the videos that had been recorded of the modeling process.

Figure 15 shows scenes of collaborative modeling. Figure 15a shows that Person B asked Person A to grasp a red brick with verbal direction and a hand gesture. Figure $15 \mathrm{~b}$ shows that Person B directed Person A where and how the red brick should be put on. Figure 15c shows that Person A followed Person B's advice and transferred the red brick.

From the videos recorded during modeling of two places, we observed these bodily actions:

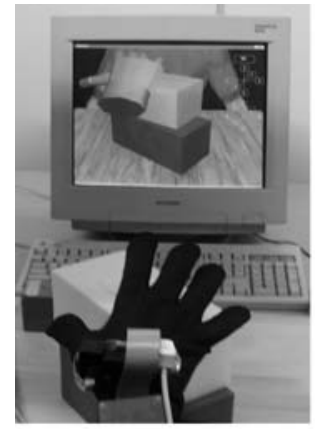

(a) Going for

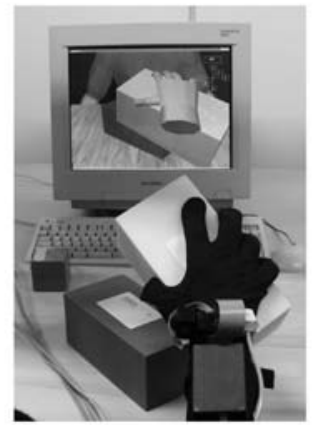

(b) Grasped

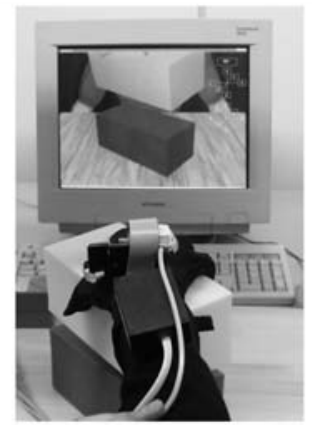

(c) Holding

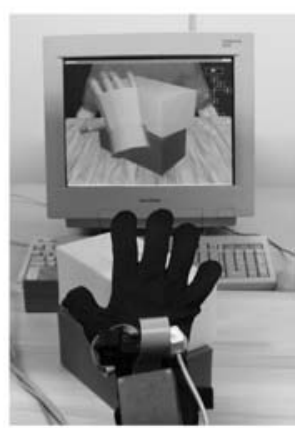

(d) Released

FIGURE 14 A scene of solo modeling with brick-reader glove. 


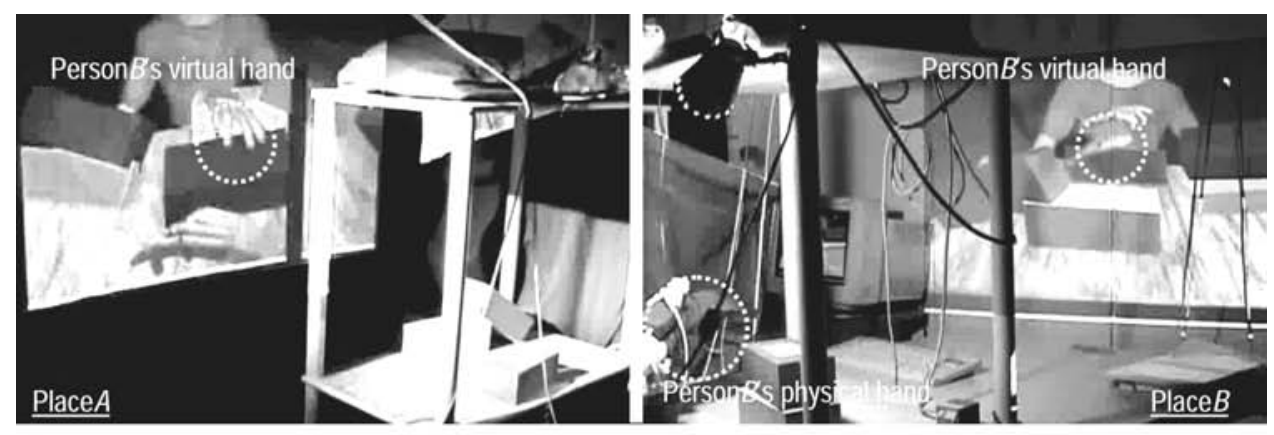

(a) $\operatorname{Person} B$ points a red brick
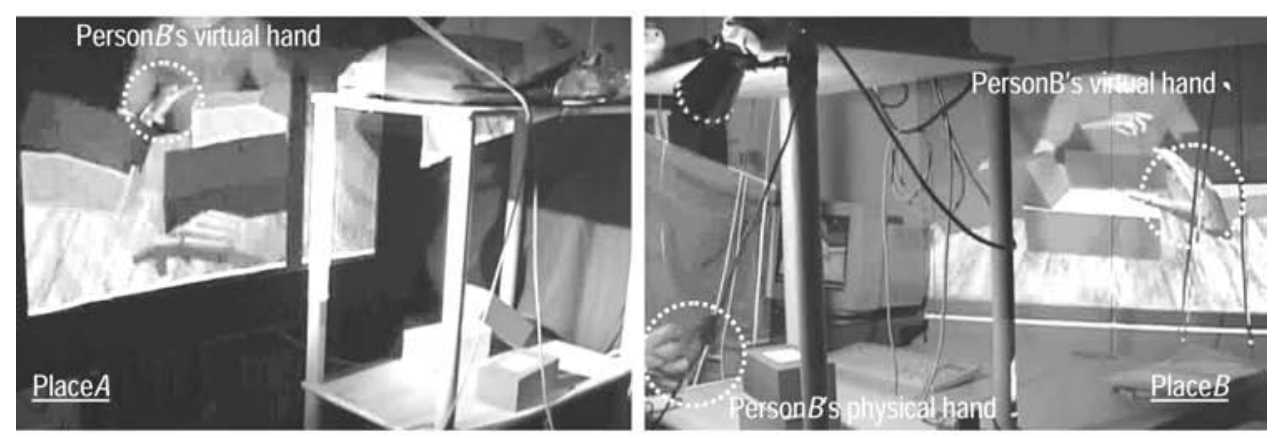

(b) Person $B$ points the positon where Person $A$ puts a red brick on

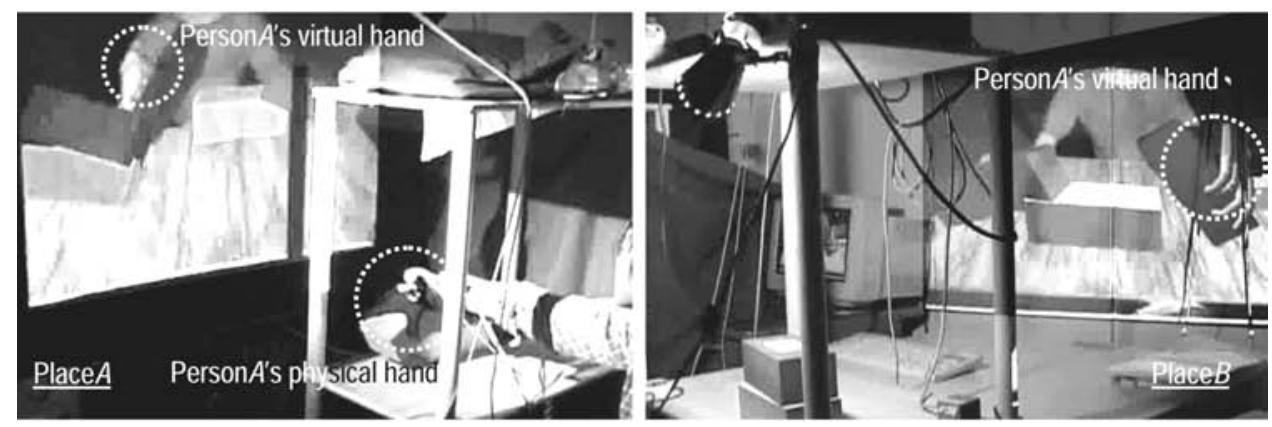

(c) Person $A$ moves the red brick

FIGURE 15 Mutual modeling with brick-reader glove 
- One person instructs a remote partner to move the specific brick accurately, and the remote partner follows the instruction.

- One person instructs a remote partner to move the brick by waving his or her virtual hand little by little when the position that he or she points to is away from the right location, and then the remote partner understands and moves the brick.

- One person instructs a remote partner how the brick should be put on by tilting his or her virtual hand.

These bodily actions and expressions observed in using the system are similar to what can be observed in collaborative modeling in a face-to-face situation.

The following list is a summary of comments obtained in interviews about the advantage of the system.

- Users can model a structure with bricks without special skills.

- Users can easily express their gestures with their hands to a remote partner.

- Users can put a virtual brick on another virtual brick just in the virtual space.

These positive comments indicate that the system can satisfy Requirement 1 by manipulating a physical brick intuitively and spatially and Requirement 2 by representing bodily actions such as spatial direction for collaborative modeling. In addition, the system can also partially satisfy Requirement 3 by interacting with the physical brick at the remote place, because the users can put a virtual brick on another virtual brick visually in a shared virtual space, although a remote physical brick cannot be manipulated directly.

Participants also gave feedback on improving the system:

- They needed to practice grasping and taking off a brick because the position of a virtual brick is sometimes different from that of the corresponding physical brick.

- They sometimes could not see gestures of a virtual avatar of a remote person and virtual bricks of remote bricks because the viewpoint in the virtual space is fixed.

- Viewpoint in the virtual space should be easily changeable for natural interaction.

- An appearance of a virtual avatar without a virtual arm seems strange.

- Eye gaze of a virtual avatar should be supported.

These results suggest that the brick-reader glove interface system can represent visual modeling with physical bricks in a virtual space and can support people, who are located in physically separate places, performing collaborative modeling in three dimensions by expressing their gestures in the common virtual space as if they were in the same place.

\section{DISCUSSION}

Integration of the brick-modeling interface system and the brick-reader glove interface system will allow remote people to construct a virtual structure with physi- 
cal bricks accurately and collaboratively. However, considering complicacy of producing a brick I/O device and the requirements of maintenance for the device, utilizing only the brick-reader glove interface system might be generally wieldy. Therefore, we investigated differences among the position and posture of a physical brick and those of a virtual brick when a user constructs a structure in three dimensions with the brick-reader glove interface system.

We measured the position and posture of a virtual brick in a virtual space when a person put a physical brick directly on another physical brick, parallel with the surface of a table. The experiment results showed that when a virtual brick was represented in the virtual space, the maximum error along $X, Y$, and $Z$ axes was approximately 20 to $30 \mathrm{~mm}$, whereas the maximum angular error around X, Y, and Z axes was approximately 10 to $20^{\circ}$. We observed that, often, when the differences between the position of a physical object and of a virtual brick exceeded the aforementioned values, the users regrasped and re-placed the physical brick.

The reason that the production of those errors should be considered to come from the positional relation of the tag antenna to the ID tag changes in comparison with the relation of the grasping calibration is because the positional relation of a physical hand to a physical brick changes a little in each case whenever the glove grasps a brick. In addition, such a change of positional relation can also occur when a person takes the tag antenna away from the ID tag vertically or laterally.

We expected that a few positional differences between virtual space and physical place would not have many adverse influences on the brick-building play because the system is intended to support not an accurate modeling but cocreative communication. However, the positional error problem should be solved by improving the calibration or including other detecting sensors.

To support cocreative communication between remote places, the embodied communication should be supported in a common communication place where each individual body exists. Most previous communication systems supporting remote collaborative work have proposed methods for supporting communication in a "virtual" or a "video" space. However, those spaces are separated from each physical place, as the word immersiveness indicates (Hindmarsh, Fraser, Heath, Benford, \& Greenhalgh, 2000). This indicates that embodied interactions with physical objects at each physical place cannot be shared between remote places.

On the other hand, several communication systems have recently proposed an approach to supporting work in a physical place, not in a closed virtual space (Feiner, MacIntyre, \& Seligmann, 1993). Gestureman et al. (2000) demonstrated that a remote instructor can give three-dimension, machine operational instructions to an operator in a physical place.

Those previous systems can support remote collaborative work just in one physical place, not in both physical places concurrently. Sharing embodied interactions in each physical place consistently is a challenging issue. Consequently, such a design method has not yet been suggested or established.

AGORA (Yamashita, Kuzuoka, Yamazaki, \& Yamazaki, 1999) is one of a few approaches that has been proposed. AGORA focuses on supporting an appropriate spatial relationship between remote people and self and bodily actions such as a gesture. The system is based on a metaphor that people are around the table, can 
project a video of a remote tabletop onto the local tabletop, and can project a video of remote people onto the screen around the table.

Another design idea, Synchronized Distributed Physical Objects, was proposed to support users operating a remote physical object with each other. Through a magnet arm mechanism under a table, PSyBench (Brave, Ishii, \& Dahley, 1998) can control synchronously the position of a remote cube on the table at both sites. In addition, Visual Telephone demonstrates that an operator can view a video of a remote table, a remote person, and a self-reflection and can operate a remote object with a bilateral manipulative robot arm (Nagashima, Seto, \& Suzuki, 1995).

Although the aforementioned communication systems can support only bodily interactions in two dimensions on the surface of the table, our proposed interface systems can support bodily interactions with physical objects in three dimensions by creating a virtual space where bodily interactions with physical bricks are visually re-created in real time and by sharing the interreal virtual space at each remote physical place. In creating an interreal virtual space and bridging remote real places, our design approach can support cocreative communication between remote places, because the interface system shows that each individual in a remote physical place bodily exists in the interreal virtual space by means that embodied interactions can be reflected in the virtual space.

Consequently, our design approach is promising, proposing a new communication mode and supporting a nonverbal mode in the research field of remote communication. In addition, we expect that our design approach can be applied to more than three physical places and in the near future can develop a communication system that will support cocreative activity in real situations in local communities.

\section{CONCLUSIONS}

To support cocreative communication between remote places, we focused on brick-building play as a typical example of cocreative communication with physical objects. In this article, we proposed a novel approach to creating an interreal virtual space reflecting embodied interactions in each physical place and bridging those remote places, and we implemented two interface systems. The experiments on collaborative modeling utilizing the interface systems clearly suggest that the systems can work well in creating a virtual space visually representing a constructing process with physical bricks and can support remote collaborative work in three dimensions in the shared virtual space, providing remote users with the sense that they were at the same place.

So far, most previous communication systems employed methods to support remote collaborative communication in just a virtual or video space independent of a bodily bonded, physical place. In contrast, the proposed system can support embodied interactions in each physical place even among remote places. This suggests that our approach is promising, supporting a new communication mode of sharing embodied interactions among remote people in each bodily bonded physical place. 
In the future, this work will investigate a design approach by focusing on the whole sense of embodied interactions to support matching the timing, conveying emotions, and sharing an ambient atmosphere with each other. Then we will work on several technical problems of sharing an interreal virtual space among more than three remote places, manipulating a physical brick at a remote place, displaying the virtual space in a physical place, and utilizing a noncontact position and posture tracking sensor. Moreover, we plan to apply the interface systems to a practical application such as tele-rehabilitation and relationship building between remote families.

\section{REFERENCES}

Brave, S., Ishii, H., \& Dahley, A. (1998). Tangible interfaces for remote collaboration and communication. Proceedings of CSCW'98 (pp. 169-178). New York: ACM Press.

Dreyfus, H. L. (2001). On the Internet (Thinking in action). New York: Routledge.

Feiner, S., MacIntyre, B., \& Seligmann, D. (1993). Knowledge-based augmented reality. Communications of the ACM, 36(7), 52-62.

Gorbet, M., Orth, M., \& Ishii, H. (1998). Triangles: Tangible interface for manipulation and exploration of digital information toporography. Proceedings of CHI'98 (pp. 49-56). New York: ACM Press.

Heath, C., \& Luff, P. (1991). Disembodied conduct: Communication through video in a multi-media office environment. Proceedings of $\mathrm{CHI}^{\prime} 91$ (pp. 99-103). New York: ACM Press.

Hindmarsh, J., Fraser, M., Heath, C., Benford, S., \& Greenhalgh, C. (2000). Object-focused interaction in collaborative virtual environments. ACM Transactions on Computer-Human Interaction, 7, 477-509.

Kasama, H. (2001). <Sunaba>-to-kodomo [TRANSLATION]. Tokyo: Toyokan.

Kitamura, Y., Itoh, Y., Masaki, T., \& Kishino, F. (2000). ActiveCube: A bi-directional user interface using cubes. Fourth International Conference on Knowledge-Based Intelligent Engineering Systems \& Allied technologies, 99-102.

Kuzuoka, H., Oyama, S., Yamazaki, K., Suzuki, K., \& Mitsuishi, M. (2000). GestureMan: A mobile robot that embodies a remote instructor's actions. Proceedings of CSCW2000 (pp. 155-162). New York: ACM Press.

Miwa, Y. (2001). Communication technology of "Ba" in co-creation. Systems/Control/Information, 45-11, 638-644.

Miwa, Y., Wesugi, S., Ishibiki, C., \& Itai, S. (2001). Embodied interface for emergence and co-share of 'Ba.' Usability Evaluation and Interface Design, 1, 248-252.

Naef, K., \& Kashiwagi, H. (2000). naef Design. Kyoto, Japan: Seigensha.

Nagashima, Y., Seto, M., \& Suzuki, G. (1995). Remote collaboration support system employing action media. Transactions of IEICE Transactions, J78-D-II(1), 115-123.

Nakai, T., Tadenuma, M., Tanaka, S., \& Nakao, K. (2001). Cypher: Cyber photographer in wonder space. Proceeding of 1st Conference on Computational Semiotics for Games and New Media (pp. 27-30).

Poupyrev, I., Tan, D., Billinghurst, M., Kato, H., Regenbrecht, H., \& Tetsutani, N. (2002). Developing a generic augmented-reality interface. IEEE Computer, 35(3), 44-50.

Rekimoto, J., Ullmer, B., \& Oba, H. (2001). DataTiles: A modular platform for mixed physical and graphical interactions. Proceedings of CHI2001 (pp. 269-276). New York: ACM Press. 
Schäfer, K., Brauer, V., \& Bruns, W. (1997). A new approach to human-computer interaction-Synchronous modelling in real and virtual spaces. Proceedings of DIS'97 (pp. 335-344). New York: ACM Press.

Shimizu, H., Kume, T., Miwa, Y., \& Miyake, Y. (2000). Ba and co-creation. Tokyo, Japan: NTT Publishing.

Tang, J. C., \& Leifer, L. J. (1988). A framework for understanding the workspace activity of design teams. Proceedings of CSCW'88 (pp. 244-249). New York: ACM Press.

Want, R., Fishkin, K., Harrison, B., \& Gujar, A. (1999). Bridging real and virtual worlds with electronic tags. Proceedings of CHI'99 (pp. 370-377). New York: ACM Press.

Wesugi, S., Itai, S., \& Miwa, Y. (2001). Hands-on modelling interface system for co-creative telecommunication. Proceedings of INTERACT2001 (pp. 675-677). Amsterdam, The Netherlands: IOS Press.

Yamashita, J., Kuzuoka, H., Yamazaki, K., \& Yamazaki, A. (1999). AGORA: Supporting multi-participant telecollaboration. Proceedings of HCII'99, 2, 543-547. 